\title{
Research Paper \\ Designing a Gait Enhancer and Determining its Effect on Standing Ability and Gait Speed of Children With Cerebral Palsy Spastic Diplegia
}

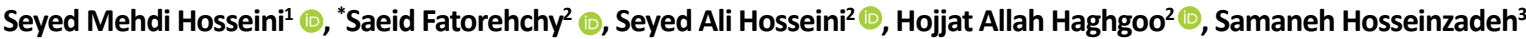

1. Department of Mechanical Engineering, Toosi University of Technology, Tehran, Iran.

2. Department of Occupational Therapy, University of Social Welfare and Rehabilitation Sciences, Tehran, Iran

3. Department of Biostatics, University of Social Welfare and Rehabilitation Sciences, Tehran, Iran.

\begin{tabular}{|c|c|}
\hline $\begin{array}{l}\text { Use your device to scan } \\
\text { and read the article online }\end{array}$ & \\
\hline aprista & $\begin{array}{l}\text { mining its Effect on Standing Ability and Gait Speed of Children With Cerebral Palsy Spastic Diplegia (Persian)]. Archives of } \\
\text { Rehabilitation. 2021; 21(4):436-453. https://doi.org/10.32598/RJ.21.4.542.1 } \\
\text { dol'https://doi.org/10.32598/RJ.21.4.542.1 }\end{array}$ \\
\hline
\end{tabular}

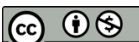

Received: 04 Mar 2019 Accepted: 20 Jan 2020 Available Online: 01 Jan 2021

Keywords: Cerebral palsy, Gait training, Standing ability, Gait speed

\section{A B STRACT}

Objective This study aimed to design a "gait enhancer" and investigate its effect on standing ability and gait speed of children with cerebral palsy spastic diplegia.

Materials \& Methods A new gate trainer was designed based on Theo Johnson mechanism. Johnson's two separate movement chains were placed on either side of the gate trainer body and attached to the lower limbs by a foot plate. To investigate the effect of the designed device, a single-item experimental study with baseline design, treatment and maintenance (ABA) was performed on four children with available spastic diplegia cerebral palsy. These children received routine occupational therapy sessions. Results The designed "gait enhancer" increased standing ability and gait speed scores in all subjects. Non-overlapping measures also indicated the improvement in both variables. Measured by Cohen's $d$, the effect size for standing ability were 1.95, 2.29, 1.83, and 2.3 for the child No. 1, 2, 3, and 4, respectively. Regarding walking speed, the effect size for these children, No. 1 to 4 , were $1.13,3.37,2.15$, and 2.21 , respectively. Cohen's d values were greater than 0.8 , indicating the considerable effect of the intervention. Hedges' $g$ was also calculated due to the small sample size, which was greater than 0.8 for all subjects in standing ability and gait speed.

Conclusion Following the use of Gait Enhancer along with conventional occupational therapy, we observed an increase in the ability to stand and walk at children with cerebral palsy. Findings showed that the change in standing ability and walking speed occurred more during the period of using the designed device than other stages, which could be a consequence of using Gait Enhancer along with routine occupational therapy sessions at this stage of the study. However, it should be noted that this study was only a single case study and to prove the effectiveness of this tool in children with cerebral palsy, it is necessary to conduct clinical trial studies.

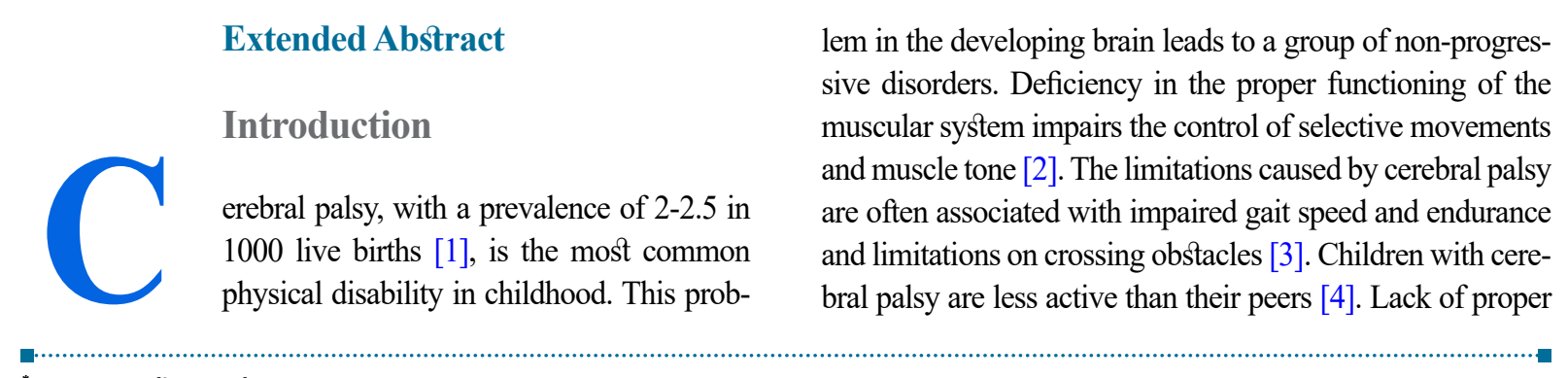

\section{${ }^{*}$ Corresponding Author:}

Saeid Fatorehchy, PhD.

Address: Department of Occupational Therapy, University of Social Welfare and Rehabilitation Sciences, Tehran, Iran.

Tel: +98 (21) 22180037

E-Mail: saeidfatorehchy@yahoo.com 
mobility and high dependence postpones their growth and social interactions $[5,6]$. For these children and their families, improving the walking ability is the ultimate goal of rehabilitation [7] because walking plays an undeniable role in daily living activities and improving bone density and cardiopulmonary function [8].

Various studies on children with cerebral palsy have indicated a clear relationship between the severity of cerebral palsy and walking ability [9]. The severity of cerebral palsy is divided into 5 levels based on the Gross Motor Function Classification System (GMFCS) [10, 11]. Many children with cerebral palsy, especially spastic diplegia, have serious problems due to poor control of the trunk, abnormal muscle tone, and lack of coordination in the lower limbs [12]. According to the GMFCS, children at level III need to use a hand-held mobility device such as a cane to walk [13]. Half of the parents of these children admitted that the use of these devices had a good effect on their child's mobility [14]. In a study on adults with cerebral palsy, it was found that $35 \%$ of these people, despite the use of assistive devices, experienced a decrease in walking ability. In $9 \%$ of cases, this ability was completely lost over time [13]. Decreased endurance and muscle strength seem to be the main reason for this problem [15]. Gait trainers and support walkers are the most used tools by this group, and this issue has been addressed in the International Classification of Functioning, Disability, and Health [16]. However, these devices are often not used to train walking but increase the child's activity and participation [17]. Therefore, existing gait trainers are not used as tools to improve gait [17]. Although the role of gate trainers in increasing children's ability to travel further distances has been confirmed in some studies, more research is needed to investigate the overall impact of these tools [18]. Therefore, proper tools should be designed that can improve the gait of children with cerebral palsy.

\section{Materials and Methods}

The device designed in this study, "gait enhancer" was made according to Theo Jansen's mechanism. The schematic view of this mechanism is shown in Figure 1. It is known among robot designers for its adjustable design, optimal energy consumption, and fast walking pattern [19]. The device was made of a 3-mm steel sheet and aluminum bars (Figure 2). In this study, an experimental singlesubject approach with an ABA design was used. This type of research is powerful for clinical decision-making [20]. Because of the novelty of the study and the uncertainty of the appropriate effect of the designed tool, 4 children with spastic diplegia were selected from rehabilitation centers affiliated to Iran Medical Council in Tehran in 2018. The inclusion criteria for them were as follows: having cerebral palsy spastic diplegia, being 6-10 years old, walking independently with a walker, understanding and following simple verbal instructions based on the SPARCLE Questionnaire [21], being at level III based on GMFCS for cerebral palsy, having family consent to participate in the study, and lacking orthopedic surgery or Botox injections in the past year. The exclusion criteria were as follows: Having uncontrolled seizures, having dislocation or partial dislocation of the hip joint, and suffering from shortness of more than 2 $\mathrm{cm}$ in one of the lower limbs. The mean age of the participants was 7 years and 9 months, and they were all boys.

The baseline period was 4 weeks and the intervention and follow-up periods were 8 weeks. All participants had 3 routine occupational therapy sessions per week during the study. During the intervention period, besides routine occupational therapy, they practiced with the "gait enhancer" for 30 minutes 3 times per week. In most studies related to children's gait training tools, 30-40 minutes of walking with the device have been applied [8]. The gross motor function and gait speed in children were assessed with the gross motor function measure-66 (GMFM-66) and 10-m walk test, respectively. All assessments were performed without placing the child in the device. The GMFM-66 has five dimensions of lying and rolling, sitting, crawling and kneeling, standing and walking, running, and jumping [22]. In this study, only the standing dimension was investigated. The assessments were performed by a senior occupational therapist, who was blind to the study process. The 10-m walk test is a valid test for children with cerebral palsy [23]. This test is a suitable tool for measuring the results of treatment after therapeutic intervention [24]. The person is asked to travel $10 \mathrm{~m}$ with or without an assistive device and at a maximum selected speed [25].

\section{Results}

The results of single-case studies are reported and analyzed mostly in the form of graphs [26]. In this study, for each variable, graph analysis and calculation of non-overlapping indices (PND, PAND, NAP, PEM, IRD, Phi, Tau$\mathrm{U})$ were performed. The intervention effect was calculated by using Cohen's d and Hedges' g:

$$
\text { Cohen's } s^{\circ}=\left(M 2^{\circ}-M 1^{\circ}\right) / S D p o o l e d
$$

\section{Standing ability}

The assessment of children's ability to stand based on the GMFM-66 is shown in Figure 3. Based on the visual analy- 


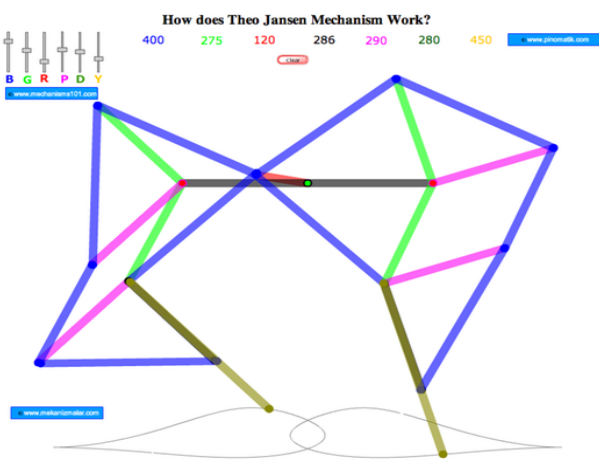

Archives of Figure 1. Schematic diagram of the Theo Jansen linkage mechanism

sis of the change process, the scores related to the standing ability in the intervention phase had an upward trend in all four children compared to the baseline scores. The standing ability of child No.1 increased by $20.5 \%$ in the intervention phase and $2.6 \%$ in the follow-up phase. In child No.2, the increase was $25 \%$ in the intervention phase and $2.7 \%$ in the follow-up phase. In child No.3, the increase was $17.9 \%$ in the intervention phase and $2.6 \%$ in the follow-up phase. In child No.4, the increase was $20.5 \%$ in the intervention phase and $7.7 \%$ in the follow-up phase.

To accurately examine the observed changes and conduct a pairwise comparison of the results between baseline and intervention phases, non-overlapping parameters were calculated. The results are given in Table 1. Comparing the baseline and intervention phases, these values were slightly lower in the third child than in the other children, but in the other three children, the obtained values indicate a further increase in scores in the intervention phase. These differences were significant. The effect size of the intervention using Cohen's d and Hedges' $g$ are presented in Table 2.

Walking ability

The results of the 10-m walk test are shown in Figure 4 for all four children. The visual analysis of the graphs shows
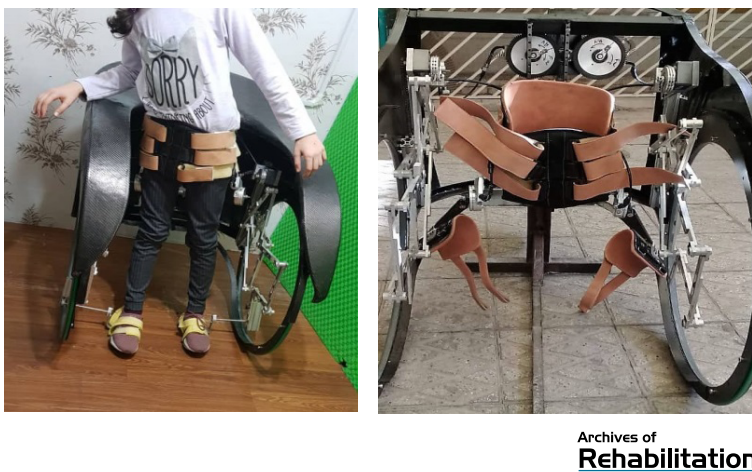

Figure 2. The designed "gait enhancer" device

the downward trend of the scores in the intervention phase. The downward trend was stopped in the follow-up phase. In Child No.1, the test duration was reduced from 11 to 8.9 $\mathrm{s}$ after the intervention, but after removing the intervention effect, this time was extended by $0.5 \mathrm{~s}$. In child No.2, there was a decrease of $3.6 \mathrm{~s}$ in the test duration following the intervention and remained constant in the follow-up phase. In child No.3 and No.4, the test times were reduced by 6 and $3.7 \mathrm{~s}$, respectively, in the intervention phase. In the followup phase for child No.3, there was a reduction in duration by $1 \mathrm{~s}$, but for child No.4, the travel time increased by $0.2 \mathrm{~s}$. Therefore, in all children, gait speed increased significantly after the intervention, but in the follow-up phase, only the third child showed a slight increase in gait speed. For the pairwise comparison of the phases, non-overlapping indices were calculated (Table 3). The obtained values indicate an increase in gait speed in all children. The increase was lower in the fourth child than in other children. Nonoverlapping measures indicated a significant reduction in the time recorded for traveling the test distance for all children. The effect size of the intervention using Cohen's d and Hedges' $g$ are reported in Table 4. The results showed a significant difference in the intervention phase compared to baseline and follow-up phases. Therefore, the use of the designed device along with conventional occupational therapy was effective in increasing the gait speed of children.

Table 1. Non-overlapping measures related to the standing ability scores between baseline and intervention phases

\begin{tabular}{cccccccccc}
\hline Child & PND (\%) & PAND & NAP & PEM (\%) & IRD (\%) & Phi & TAUnovlap & Tau-U \\
\hline 1 & 100 & 1 & 1 & 100 & 100 & 1 & 1 & 0.92 \\
2 & 100 & 1 & 1 & 100 & 100 & 1 & 1 & 0.92 \\
\hline 3 & 83 & 0.87 & 0.96 & 100 & 67 & 0.67 & 0.92 & 0.83 \\
4 & 100 & 1 & 1 & 100 & 100 & 1 & 1 & 0.92 \\
\hline
\end{tabular}



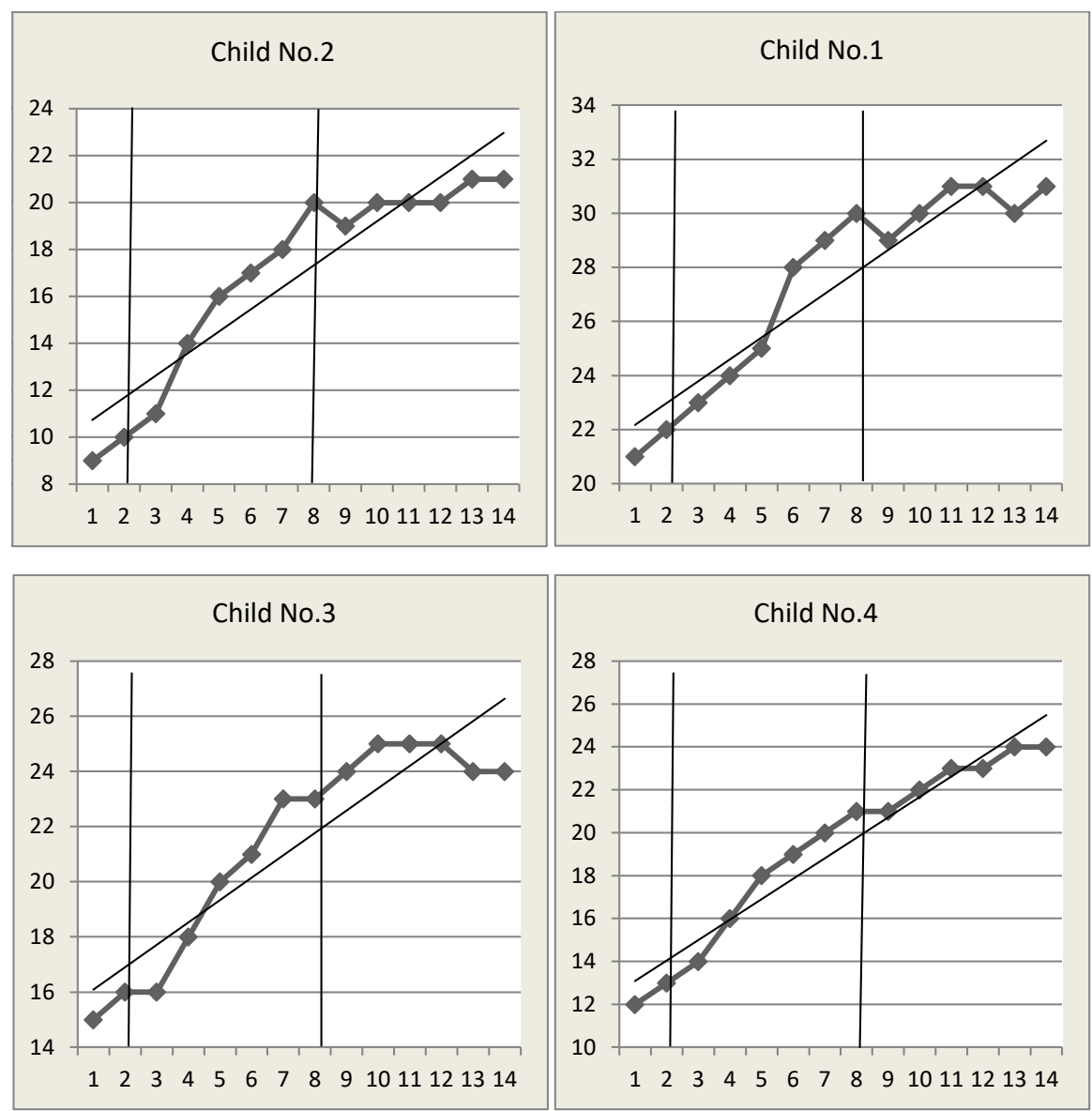

Figure 3. The standing ability scores of children with spastic diplegia based on the Gross Motor Function Measure-66

\section{Discussion and Conclusion}

The results showed the effectiveness of using the designed device in improving the standing ability of four children with spastic diplegia. This result is consistent with other study results [27, 28]. Schindl et al. investigated the effect of gait training on a treadmill in improving cerebral palsy children's standing ability. Their results showed that, although this type of intervention increased the standing ability, the rate of progress was much higher in children who could walk with weight support than in children without this ability [29]. The children in our study were all able to walk with assistive devices; hence, their results are consistent with our results. Provost et al. obtained similar results to Schindl et al. following gait training in children with cerebral palsy [30]. Because of the continuous weight-bearing exercise on the lower limbs using the gait trainer and the apparent effect of this type of exercise on increasing muscle strength and standing on the legs, an increase in the ability to stand in these children was expected [31]. By concentrating on the walking, the standing duration on two legs decreases, while its duration on one leg increases. This finding can also justify the improved standing ability in children [22]. In children No.3 and No.4, the standing duration was significantly prolonged after the intervention.

In this study, gait speed was measured using a 10-m walk test, and the results showed the desired effect of gait training with the "gait enhancer" device along with occupational

Table 2. The effect size of the intervention in improving the standing ability

\begin{tabular}{ccccc}
\hline Effect Size & Child 1 & Child 2 & Child 3 & Child 4 \\
\hline Cohen's d & 1.95 & 2.29 & 1.83 & 2.3 \\
\hline Hedges' g & 1.7 & 1.99 & 1.59 & 2 \\
\hline
\end{tabular}



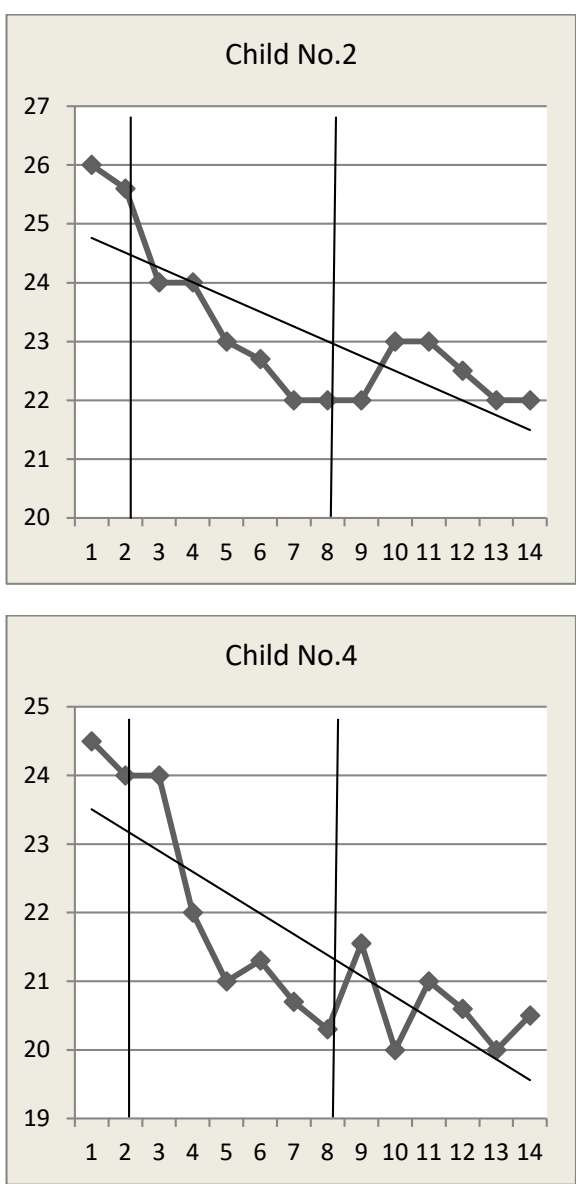

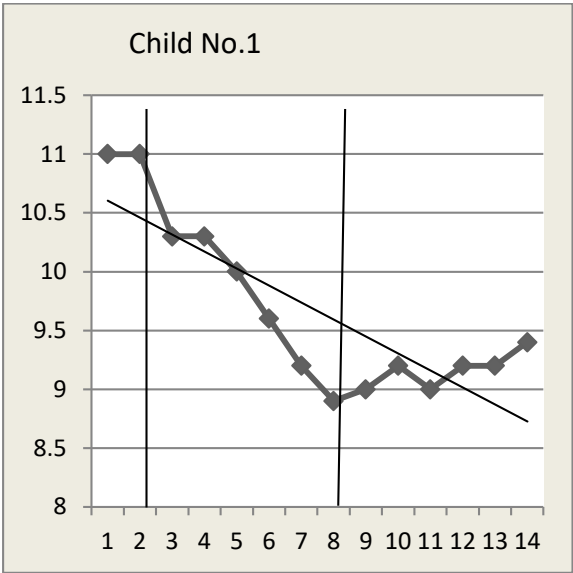

Child No.3

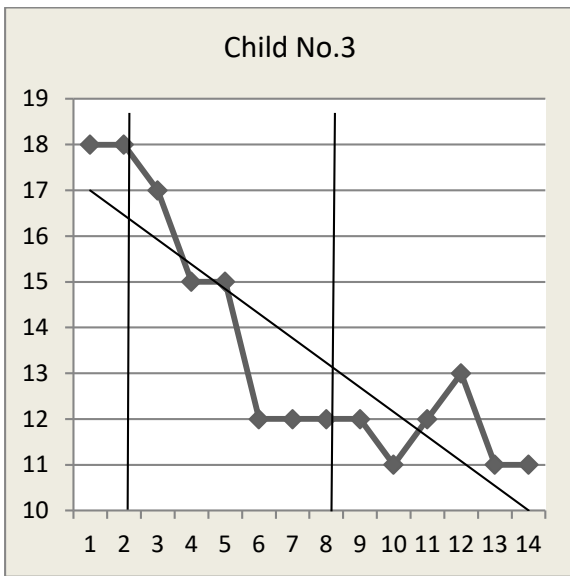

Figure 4. The recorded time (s) for children with spastic diplegia in the 10 -m walk test

therapy. Many studies have examined gait speed with the $10-\mathrm{m}$ walk test. The results of some of these studies are consistent with the results of the present study [32-34]. This similarity may be because of the reduced time it took to stand on two legs following gait training with the designed device. These assistive tools reduce the standing duration on both feet while walking by increasing the swing time in the lower limbs and, hence, increase gait speed $[35,36]$. Dodd et al. also reported improvement in gait speed after the intervention [8]. Willoughby et al. in a clinical trial, compared two ways of walking on the ground and a treadmill. Their results showed no significant differences between the two groups. Therefore, both methods increased gait speed to the same extent [37]. Fatorehchy et al. in a pilot study, examined the effect of aquatic therapy in children with cerebral palsy. The results showed improved gait balance and walking capacity in children [38]. In another study, they found an improvement in gait endurance after using gait enhancer in children with cerebral palsy [39]. Therefore, increased balance and endurance of walking can be a reason for increased gait speed in these children.

Table 3. Non-overlapping measures related to the walking ability scores between baseline and intervention phases

\begin{tabular}{ccccccccc}
\hline Child & PND (\%) & PAND & NAP & PEM (\%) & IRD (\%) & Phi & TAUnovlap & Tau-U \\
\hline 1 & 100 & 1 & 1 & 100 & 100 & 1 & 1 \\
\hline 2 & 100 & 1 & 1 & 100 & 100 & 1 & 1 & 1 \\
3 & 100 & 1 & 1 & 100 & 100 & 0.92 & 0.67 & 0.92 \\
\hline
\end{tabular}


Table 4. The effect size of the intervention in improving the walking ability

\begin{tabular}{ccccc}
\hline Effect Size & Child 1 & Child 2 & Child 3 & Child 4 \\
\hline Cohen's d & 1.13 & 3.37 & 2.15 & 2.21 \\
\hline Hedges' g & 0.98 & 2.93 & 1.87 & 1.92 \\
\hline
\end{tabular}

Although the gross motor function level of the children in the study was the same, they showed a large difference in gait performance. Therefore, only children at level III of GMFCS had to be selected for the study, and they prolonged the research process. Children have different body dimensions. This device has limited settings for a child. Another limitation of this research was the need for financial resources. In the process of designing and manufacturing the device, repeated actions were required. Moreover, difficulty in purchasing equipment and manufacturing different parts made the research process longer than initially expected. Because of the effectiveness of the designed device, it is recommended that a study be performed to increase the symmetry of lower-extremity motor function in children with hemiplegia. The use of this device in children under 6 years of age is recommended for better therapeutic results.

Using the designed gait enhancer and common occupational therapy can improve the standing ability and gait speed of children with spastic diplegia. The gait enhancer is a valuable device for gait training in these children, with no adverse effects. The difference in results between the four participating children was due to differences in lower-limb muscle strength and muscle tone. This study's results cannot be generalized to all children with cerebral palsy; hence, more research is needed.

\section{Ethical Considerations}

\section{Compliance with ethical guidelines}

This study obtained its ethical approval from the Research Ethics Committee of the University of Social Welfare and Rehabilitation Sciences (Code: IR.USWR.REC.1396.286).

Funding

This research is part of the $\mathrm{PhD}$. dissertation of second author at Department of Occupational Therapy, University of Social Welfare and Rehabilitation Sciences, Tehran, Iran.
Authors' contributions

Designing of Gait Enhancer: Saeid Fatorehchy and Seyed Mehdi Hosseini; Date analysis: Samaneh Hosseinzadeh; Editing: Saeid Fatorehchy; Revision and Supervision: Hojjat Allah Haghgoo and Seyed Mehdi Hosseini.

\section{Conflict of interest}

The authors declared no conflict of interest. 
This Page Intentionally Left Blank 


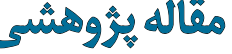
طلج مغزى و بررسى ثأثير تمرين با Gait Enhancer بر توانايى ايستادن و سرعت راه رفتن كودكان

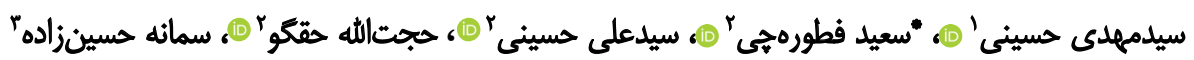

1. بروه مهندسى مكانيك، دانشكاه صنعتى خواجه نصيرالدين طوسى، تهران، ايران.

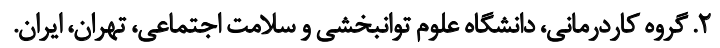

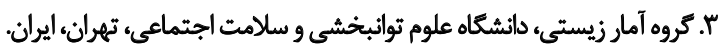

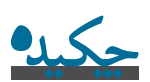

هذافت اين مطالعه به منظور طراحى و و بررسى تأثير استفاده از Gait Enhancer بر توانايع ايستادن و سرعت راه رفتن كودكان فلج مغزى صورت كرفت.

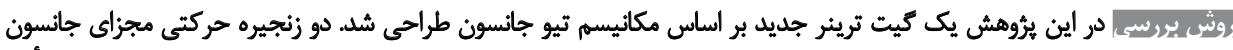

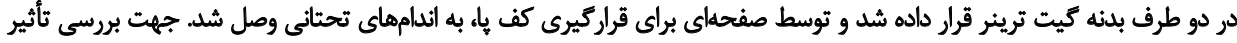

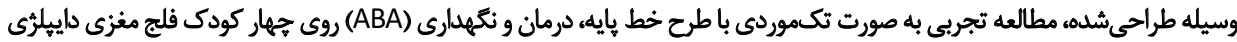

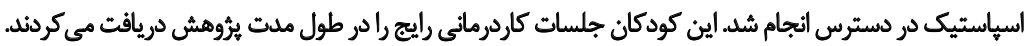

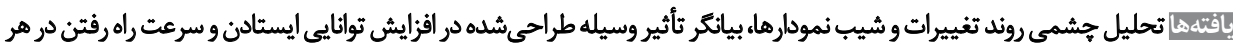

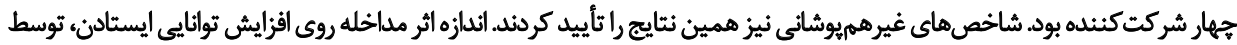

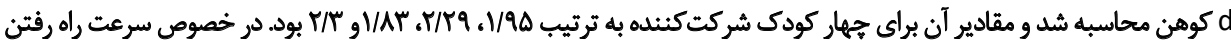

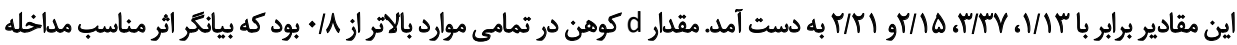

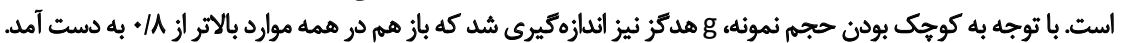

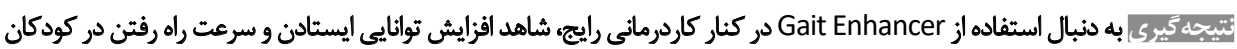

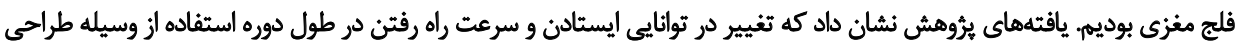

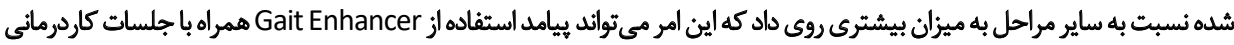

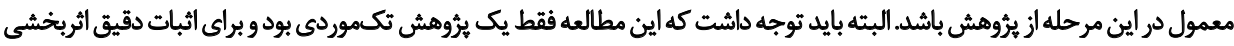
اين ابزال در كودكان فلج مغزي، نياز به انجام مطالعات كار آزمايى بالينى است.

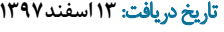

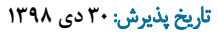

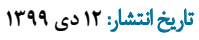

در عملكرد مناسب سيستم عضلاتي منجر به مواردى همجهون

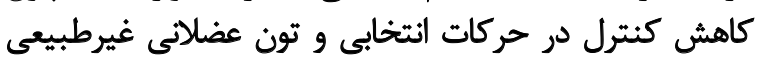

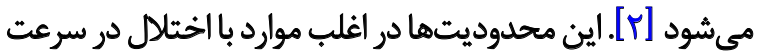

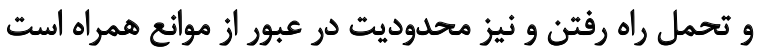

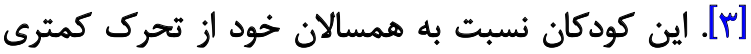

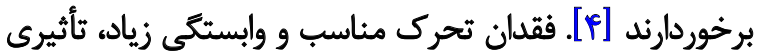

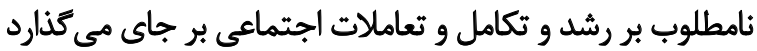

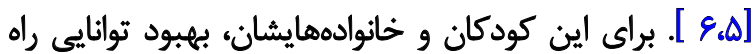

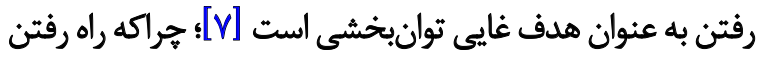

مقلمه

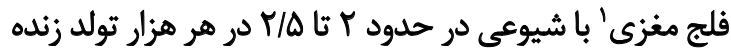

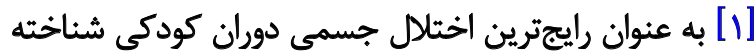

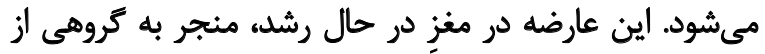

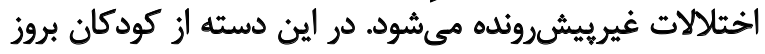

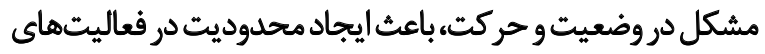
مختلف زندكى و ميزان مشاركت مي شود. از طرف ديكر نقص

1. Cerebral Palsy (CP) 
واكر بوده است؛ بنابراين نياز به طراحي و به كارئيرى ابزارى است

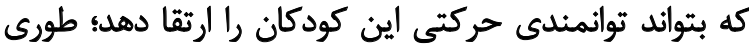

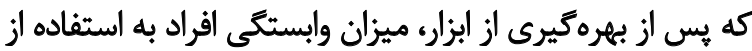

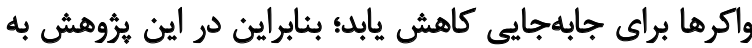

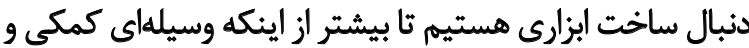

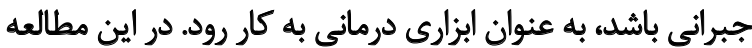

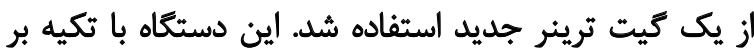

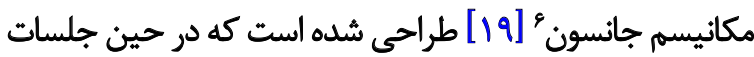

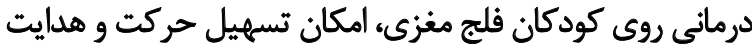

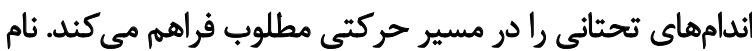
اين دستكاه Gait Enhancer استئ

$$
\text { روش بروشى }
$$

وسيله طراحى شده در اين مطالعه با تكيه بر مكانيسم جانسون

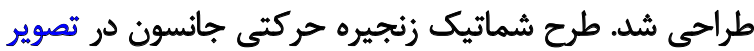

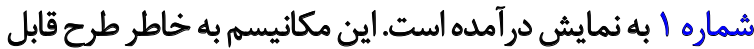

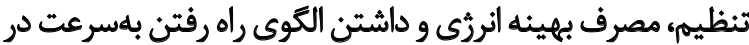

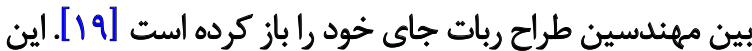

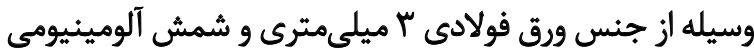

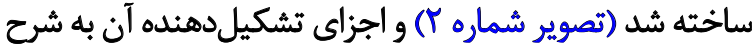
زير است (تصوير شماره ب):

1. زنجيره حركتى: دو زنجيره حركتى جانسون به شكل قابل

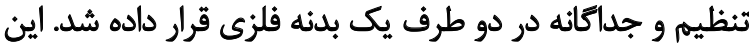

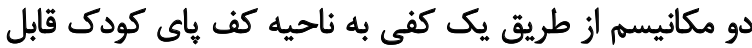

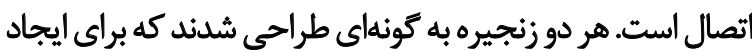

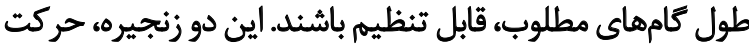

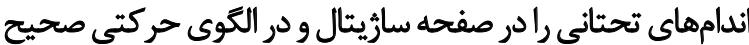

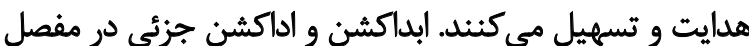

$$
\text { ران امكانيذير است. }
$$

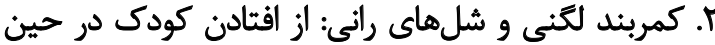

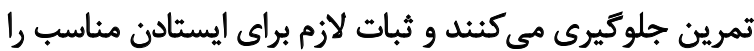

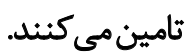

r. كابل هاى ساده: از كابل هاى ساده براى انتقال نيروى بيشر باند

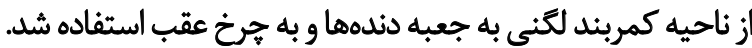

روش استفاده از وسيله: كودى به حالت ايستاده درون ابزار قرار

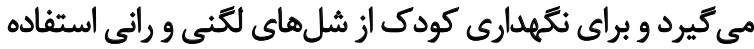

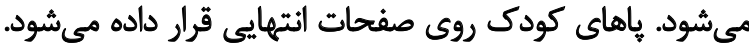

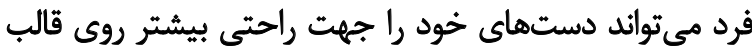

$$
\text { بيرونى بدنه ابزار بكذارد. }
$$

نقشى انكارنايذير در انجام فعاليتهاى روزمره زندكى و بهبود إنيود

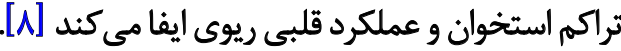

مطالعات مختلف روى كودكان فلج مغزى حاكى از وجود

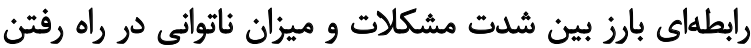

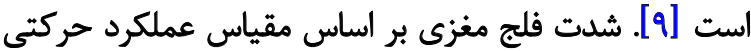

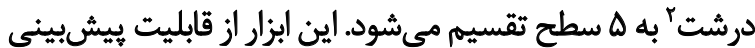

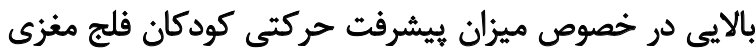

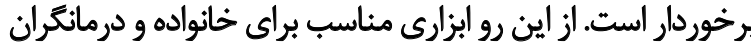

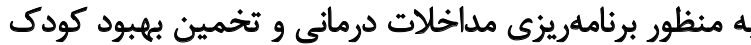

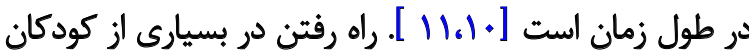

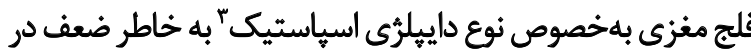

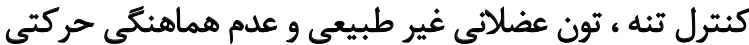

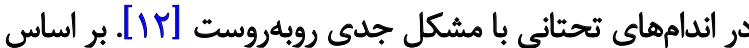

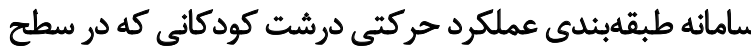
سه قرار مى كيرنده براى راه رفتن نيازمند استفاده از عصائ يا واكر كائي

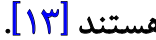

نيمى از والدين اين كودكان اذعان داشتهاند كه استفاده از اين

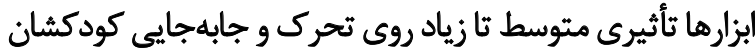

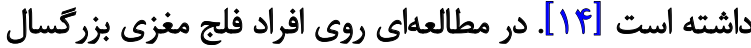

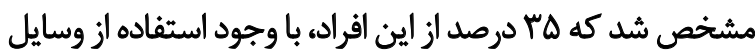

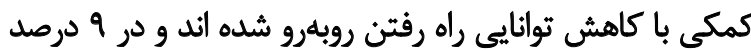

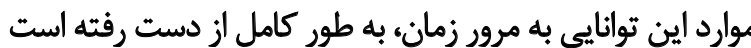

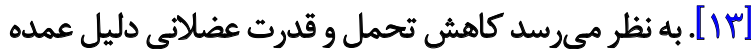

اين امر است [1ه]

كيت ترينرها و واكرهاي حمايتى بيشترين ابزارهاى مورد

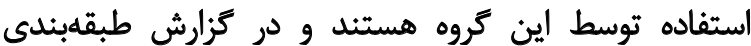

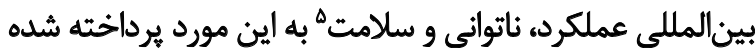

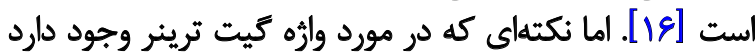

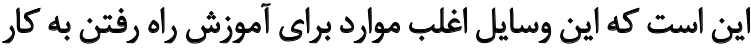

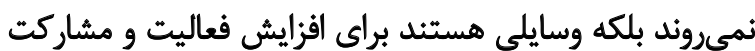

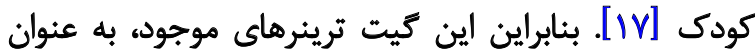

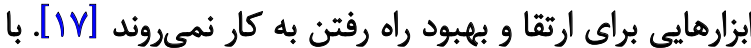

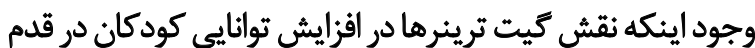

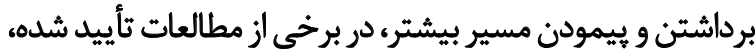

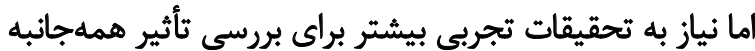

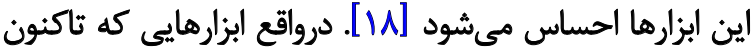

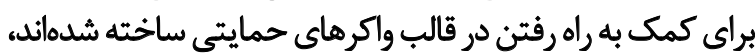

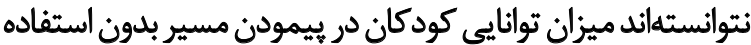
از ابزار را افزايش دهند و كودك برائ حركت هميشه وابستئه بـانه

2. Gross motor function classification system (GMFCS)

3. Spastic diplegia

4. Gait trainer

5. ICF 
بود. همه شركت كنندكان در طول مطالعه، سه جلسه كاردرمانى

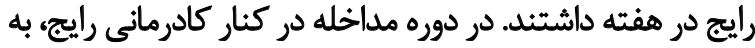

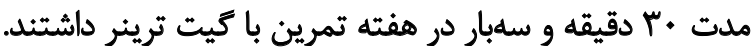

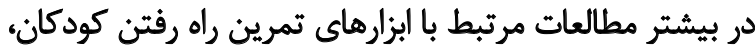

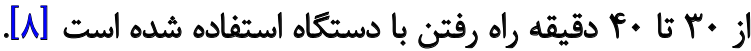

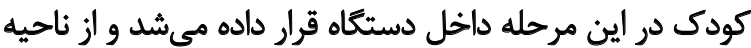

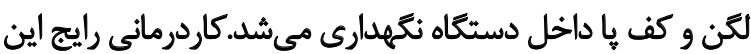
كودكان با تكيه بر تكنيكهاي بوبت و و رود و آموزش راه راه رفتن باردان واكرهاي ساده بود. أبزاوهاى الرويابي ارزيابى عملكرد حركتى درشت و سرعت راه رفتن در كودكان

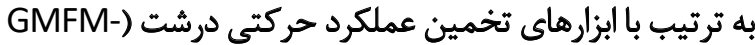

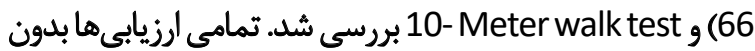

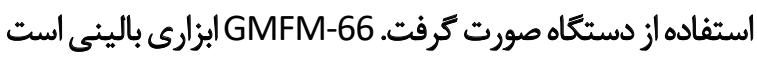

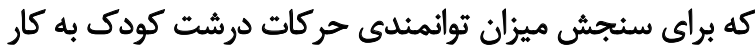

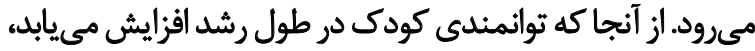

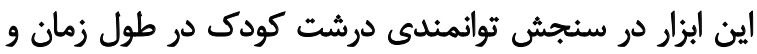

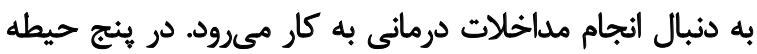

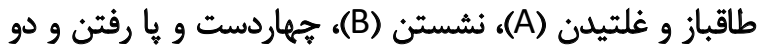

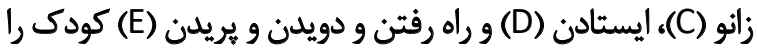

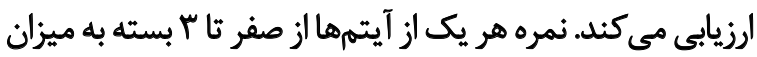

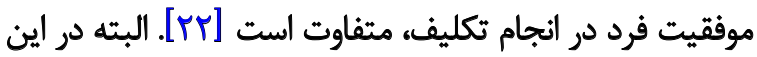

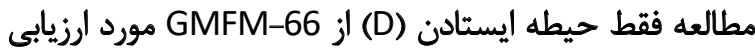

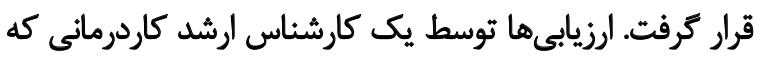
از مراحل يُؤوهش بيى اطلاع بود، صورت كرفت. test براى كودكان فلج مغزى به عنوان آزمونى داراى اعتبار شئاخته

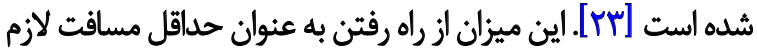

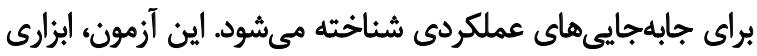

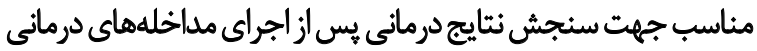

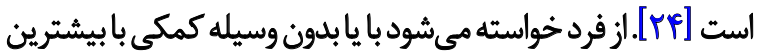

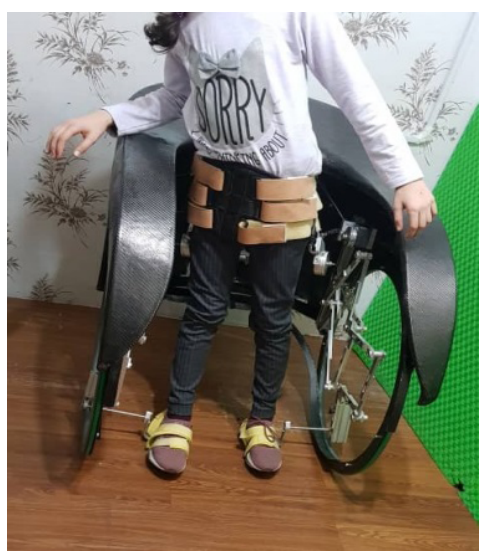

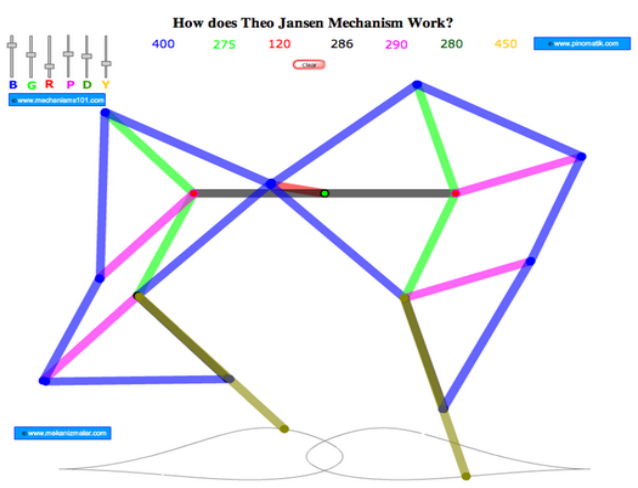

توانبخننى

$$
\text { تصوير (.نماى شماتيك از زنجيره حركتي جانسون }
$$

$$
\text { روشهاى تجزيه و تحليل آمارى وسيله طراحى }
$$

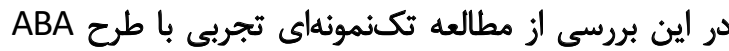

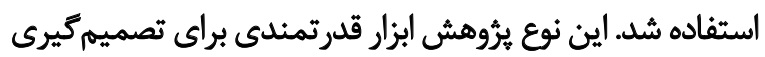

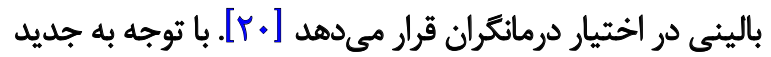

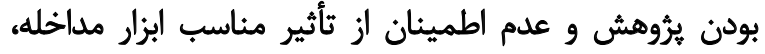

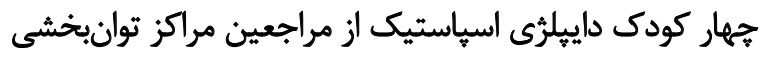

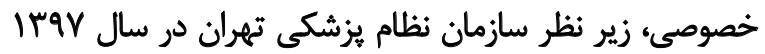

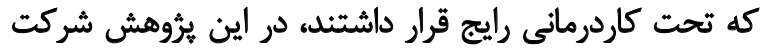

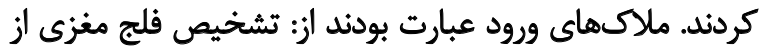

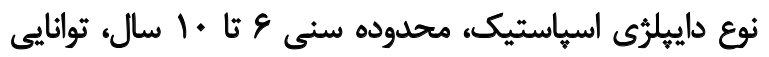

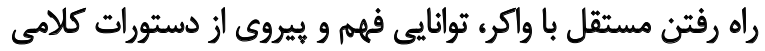

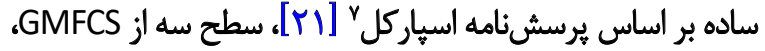

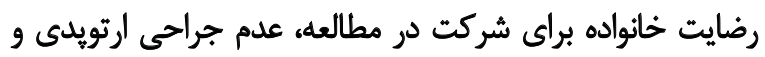

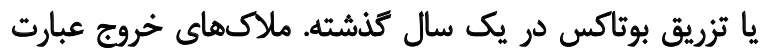

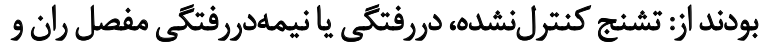

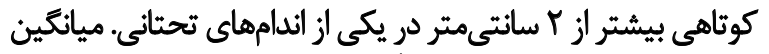

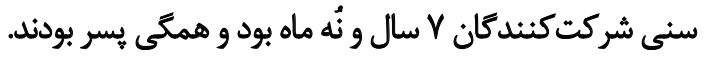
دوره يايه جهار هفته ودوره مداخله وييكيرى شامل هشت هفته

\section{SPARCLE}

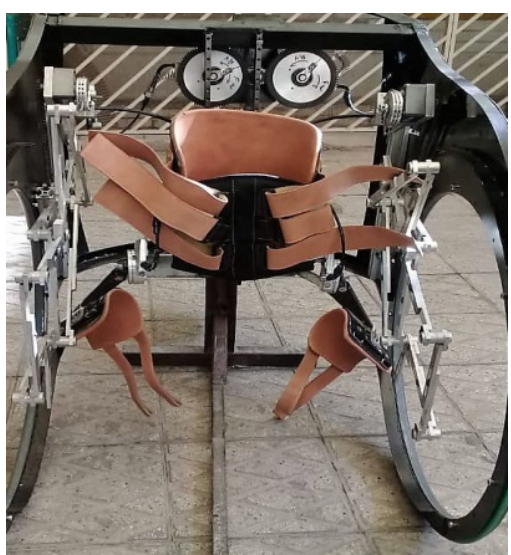

تصوير Y. مكاثيسم تقويت كثنده راه رفتن 


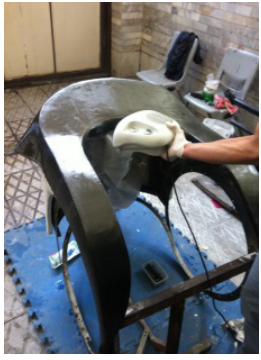

توانبخننى
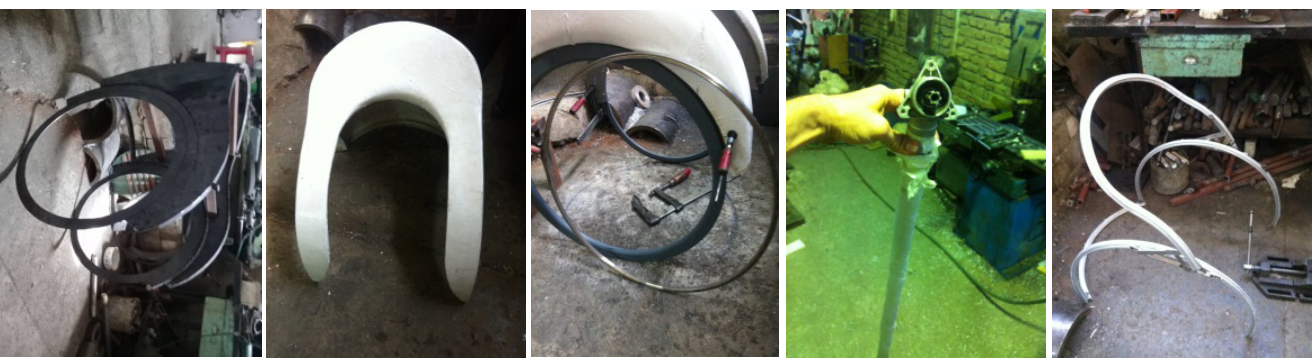

تصوير با. اجزاي مختلف دستكًاه

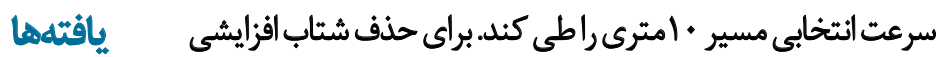

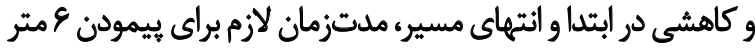
نتايج حاصل از مطالعات تكموردى در اكثر موارد به صورت

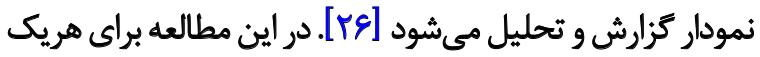

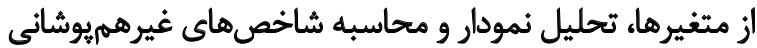
انجام شده (PND, PAND, NAP ,PEM ,IRD ,Phi , Tau-U) است. اندازه اثر مداخله توسط d كوهن و و هد أز محاسبه كرديده

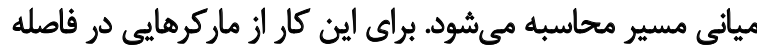

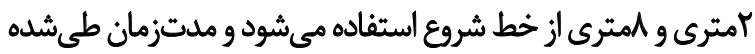

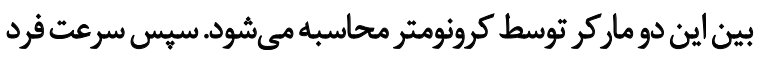

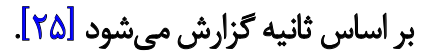
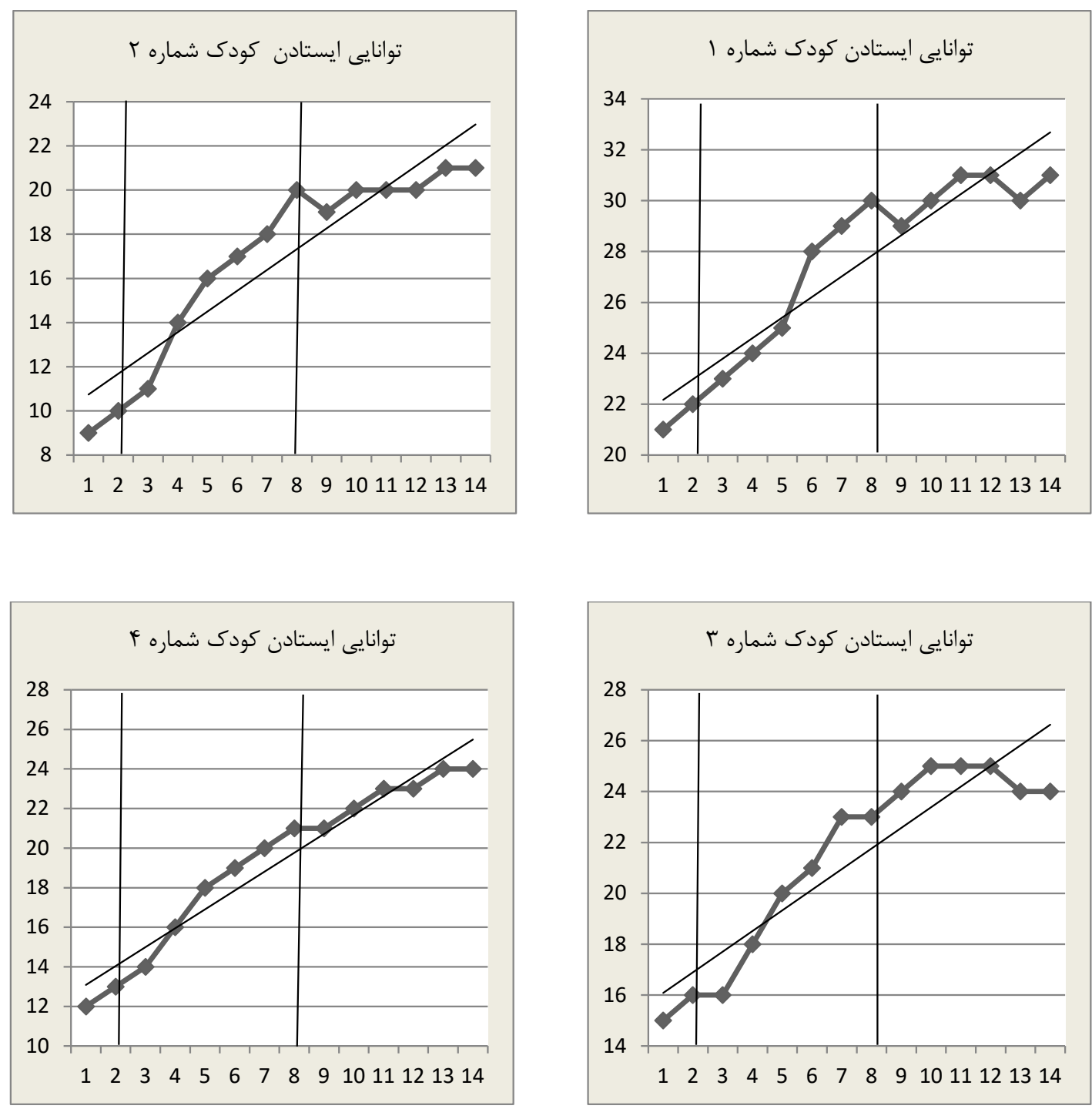

توانبخنتى

تصوير ع. نمودار نمرات ايستادن بر اساس GMFM-66 در هر جهار كودك داييرّى اسياستيك 


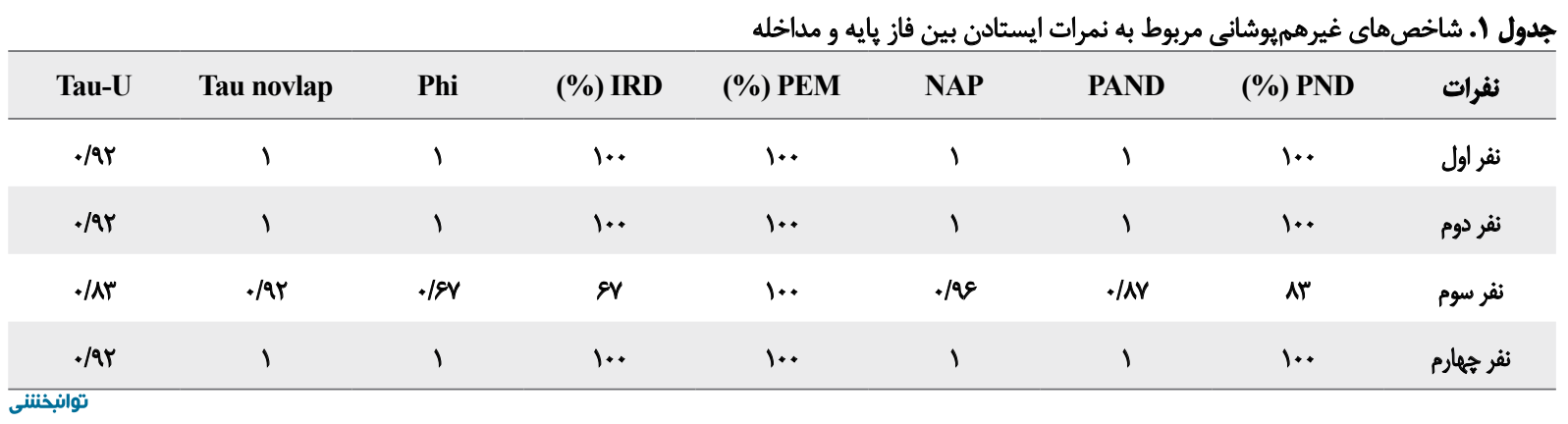

از افزايش بيشتر نمرات در فاز مداخله است. مقايسه شاخصهاى

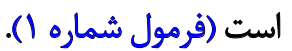

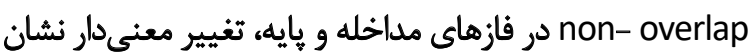

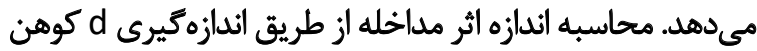

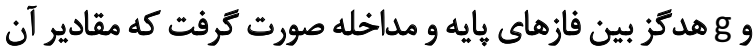

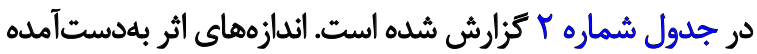
بيانكر تغيير معنى دار در توانايى ايستادن كودكان است أنتان.

با توجه به شيب نمودارها و با نكاهى بر شاخصهاى

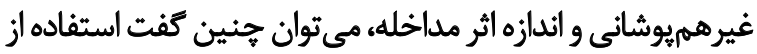
Gait trainer كودكان مطالعه مؤثر بوده است.

نتايج بررسى سرعت بيمودن مسير بر اساس

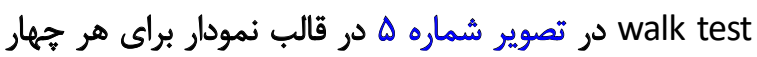

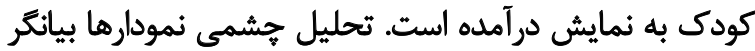

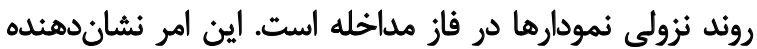

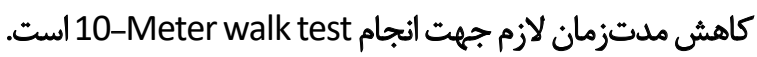

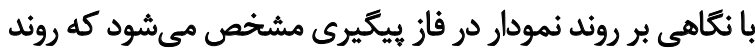

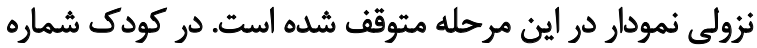

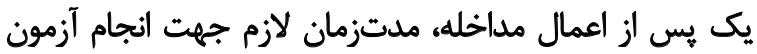

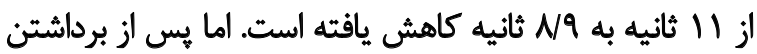

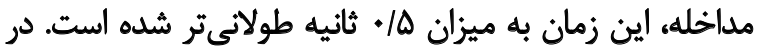

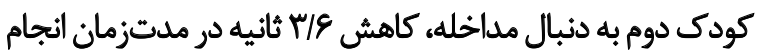

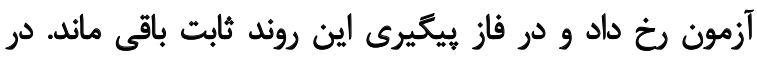

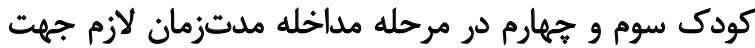

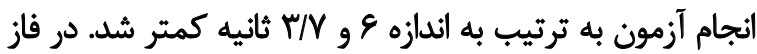

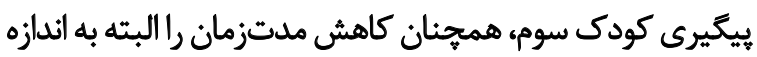

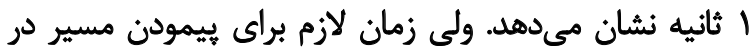

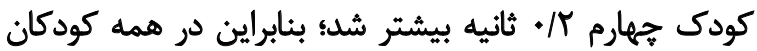

\section{Cohen' $s^{\circ} d^{\circ}=\left(M 2^{\circ}-{ }^{\circ} M 1\right)^{\circ} /{ }^{\circ} S D$ pooled}

GMFM- نتايج ارزيابى توانايى ايستادن كودكان بر اساساس

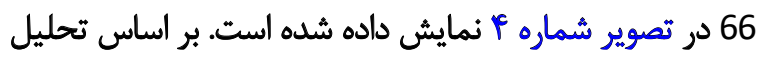

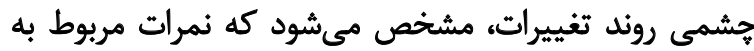

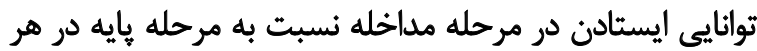

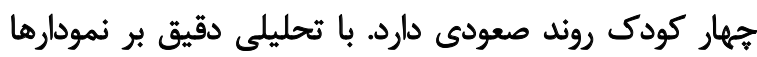

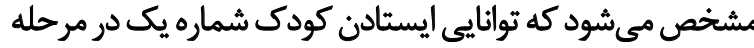

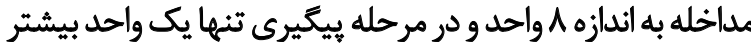

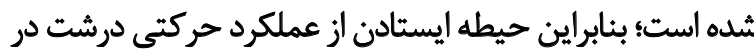

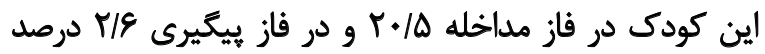

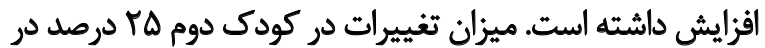

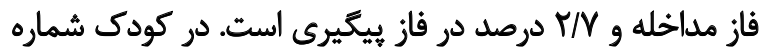

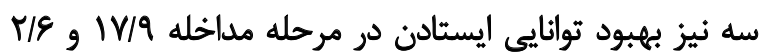

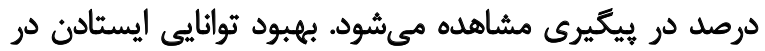

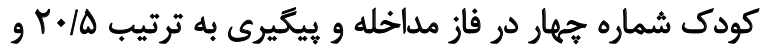

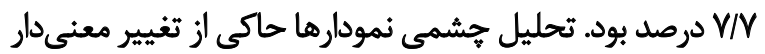

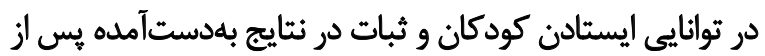
مداخله استث.

به منظور بررسى دقيق تغييرات مشاهدهشده و مقايسه فازها

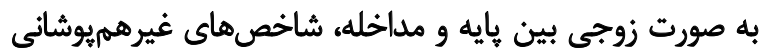

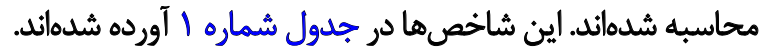
بابررسى شاخصها ملاحظه مى شود كه در مقايسه فازهاى بايه

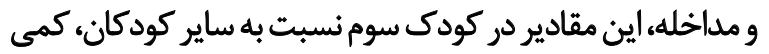
ضعيف است، ولى در سه كودى ديكر مقادير بلهدست آمده، حاكى

جدول r. اندازه اثر مداخله در افزايش توانايى ايستادن

\begin{tabular}{|c|c|c|c|c|}
\hline كودك جهارم & كودك سوم & كودكى دوم & كودى اول & اندازه اثر \\
\hline$r / m$ & V/Ar & $r / r q$ & 1/98 & Cohen's d \\
\hline$r$ & $1 / \Delta q$ & 1/99 & 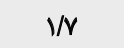 & Hedges' g \\
\hline
\end{tabular}



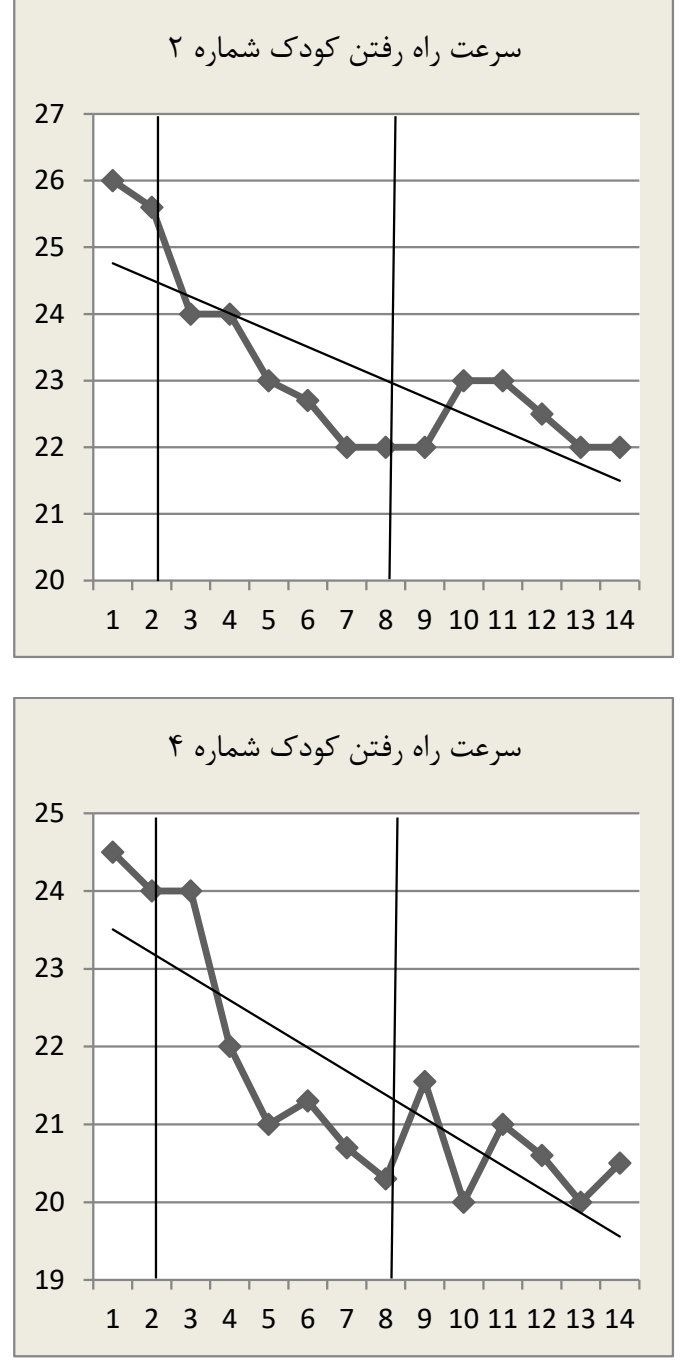

توانبخننى

شماره f به نمايش كذاشته شده است.

تحليل جشمى روند تغييرات، تفاوت قابل ملاحظهاى را در فاز

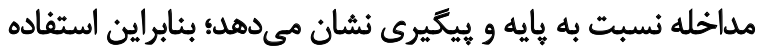

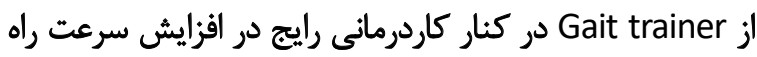

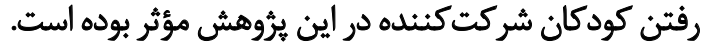
بحث

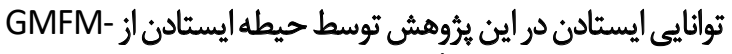

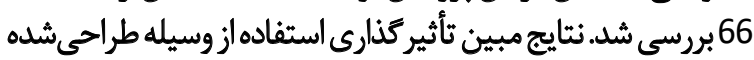

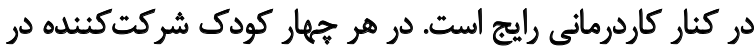

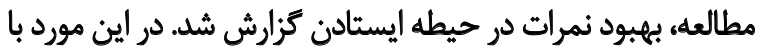

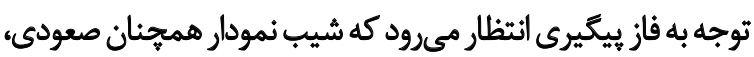

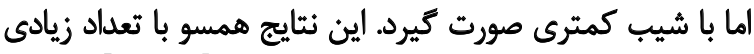

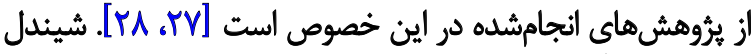

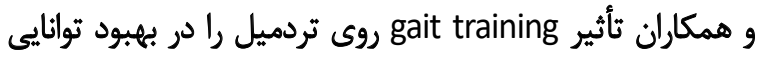
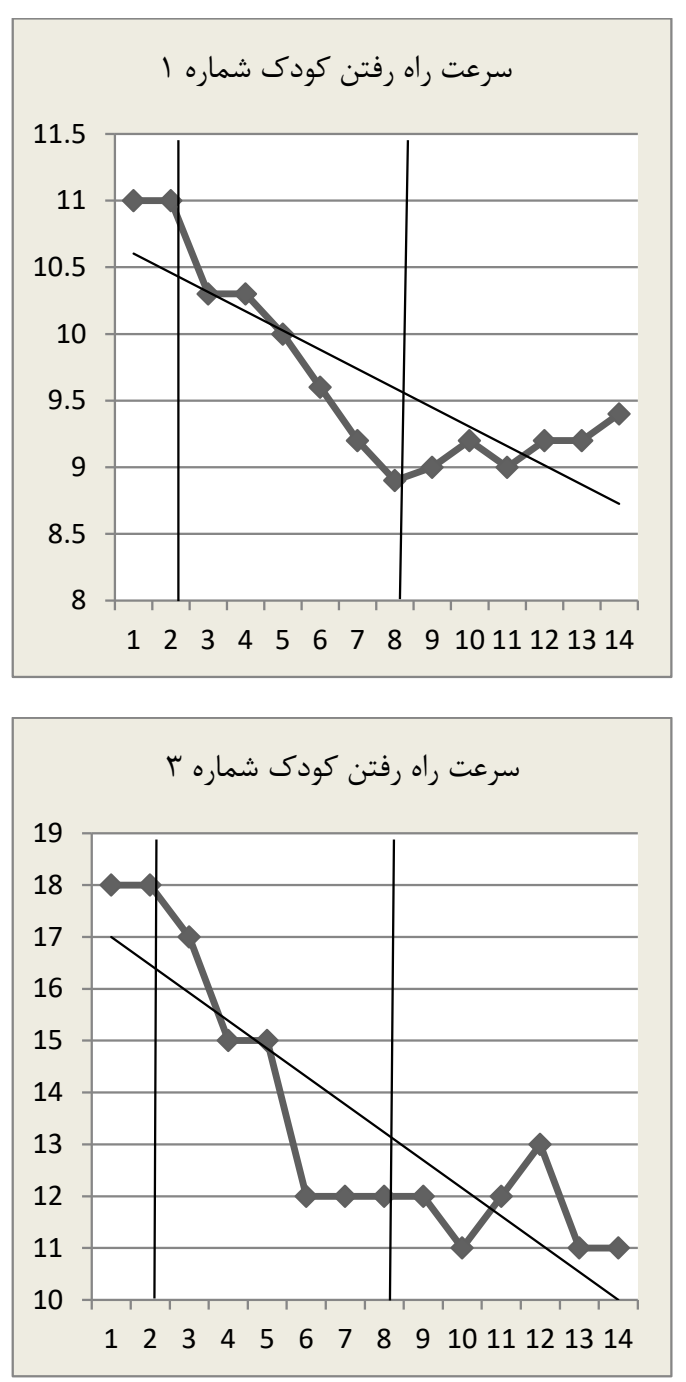

تصوير ه. نمودار مدتزمان بيمودن مسير بر اساس ثانيه

شركت كنيده در مطالعه، سرعت راه رفتن بعد از مداخله افزايش

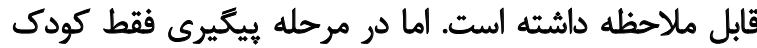
سوم، آن هم به ميزان اندكى افزايش سرعت نشان مي دهري

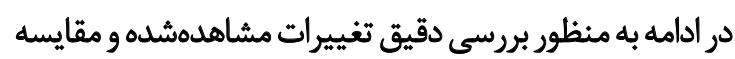

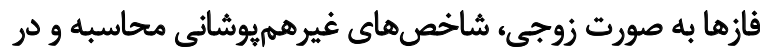
جدول شماره ب كزارش شدهاند.

وا بررسى شاخص ها ملاحظه مى شود كه در مقايسه فازهائ يايه

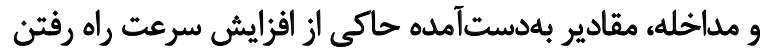

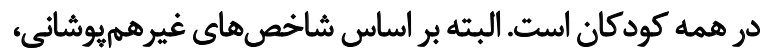

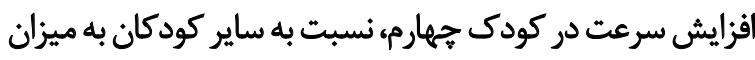

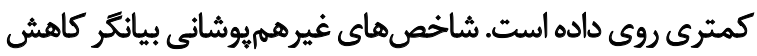

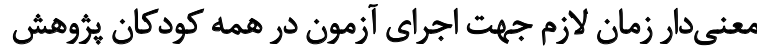

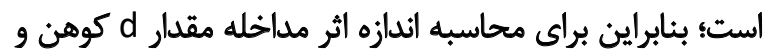

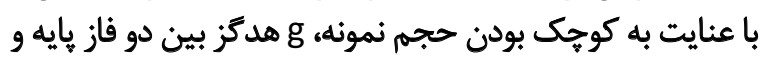

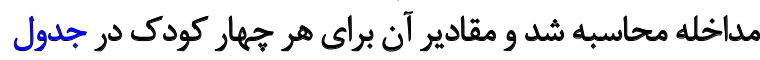


جدول "ا. شاخصهاي غيرهميوشانى مربوط به مدتزمان طى مسير بين فاز بايه و مداخله

\begin{tabular}{|c|c|c|c|c|c|c|c|c|}
\hline Tau-U & Tau novlap & Phi & (\%) IRD & (\%) PEM & NAP & PAND & (\%) PND & نفرات \\
\hline 1 & 1 & 1 & $1 .$. & $1 .$. & 1 & 1 & $1 .$. & نفر اول \\
\hline •/9Y & 1 & 1 & $1+0$ & $1+0$ & 1 & 1 & $1+0$ & نقر دوم \\
\hline 1 & 1 & 1 & $1 \ldots$ & 1.0 & 1 & 1 & $1 .$. & نفر سوم \\
\hline . & ./9r & $.18 \mathrm{~V}$ & or & $1 \ldots$ &.$/ 98$ &.$/ A Y$ & Ar & نفر جهارم \\
\hline
\end{tabular}

gait training داخل ابزار اشاره كرد؛ جراكه اين دسته از ابزارها

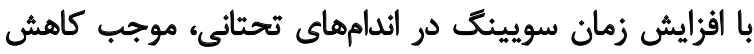

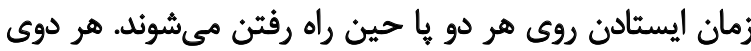

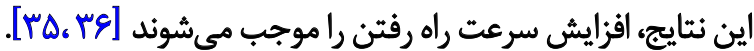

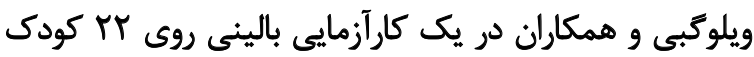

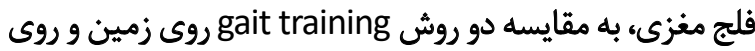

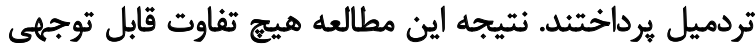

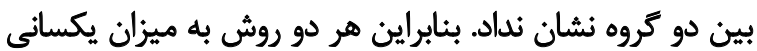

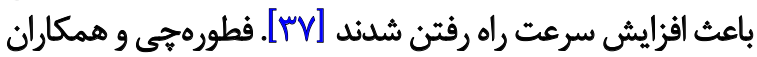

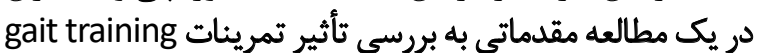

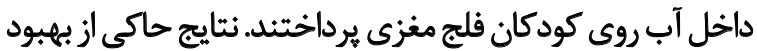

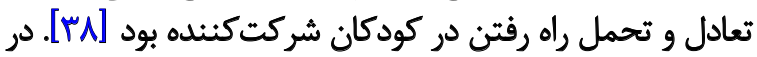

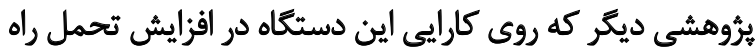

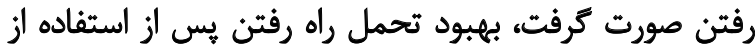

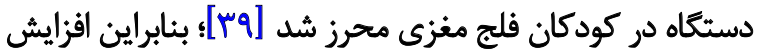

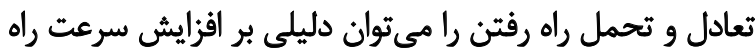

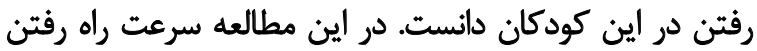

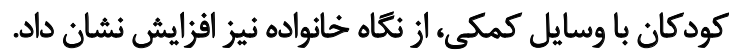

تثيجمكيرى

استفاده از اين ابزار جديد در كنار كاردرمانى رايج، مي تواند

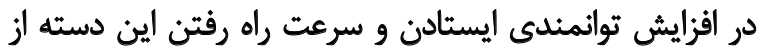

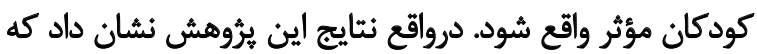

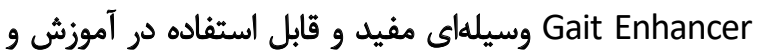

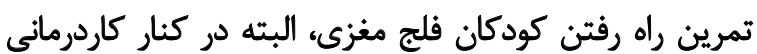

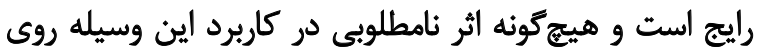

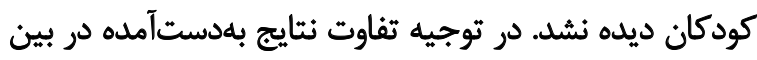

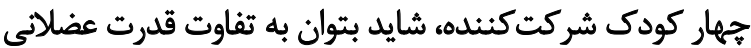

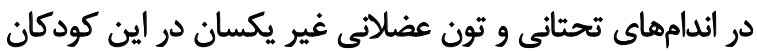

ايستادن در كودكان فلج مغزى بررسى كردند. نتايج مطالعه شيندل

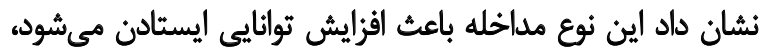

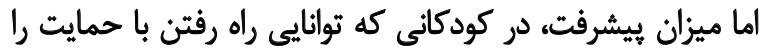

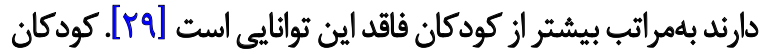

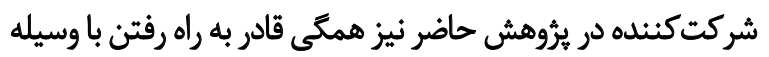

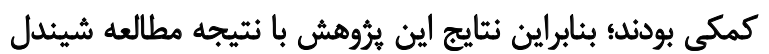

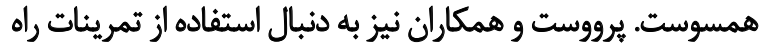

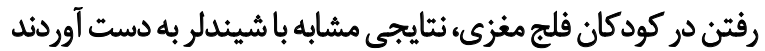

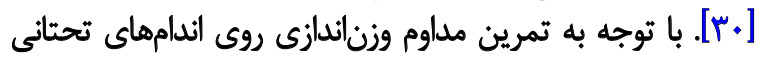

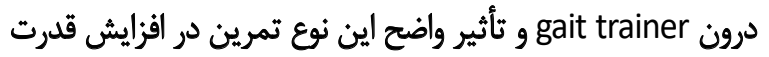

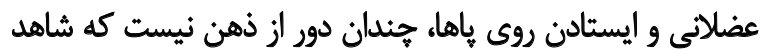

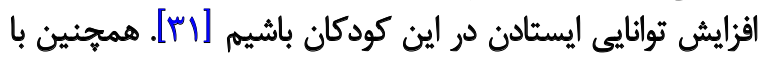

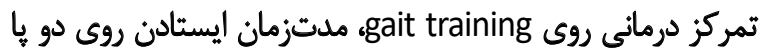

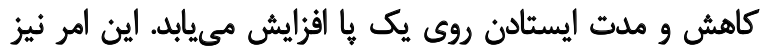

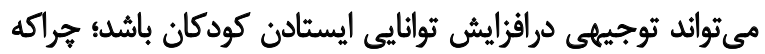

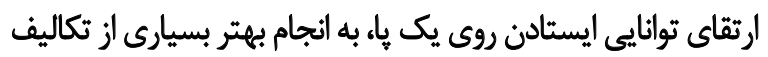

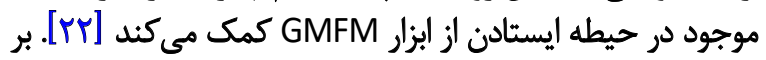

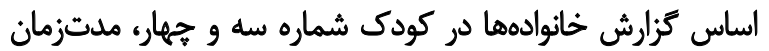

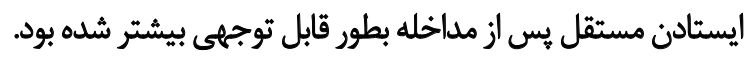

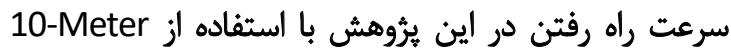

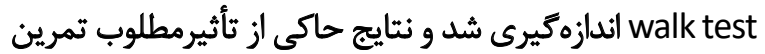

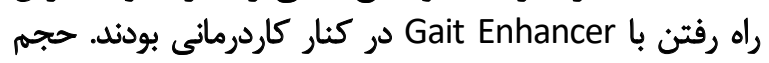

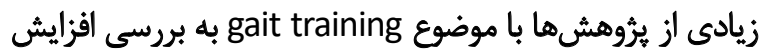

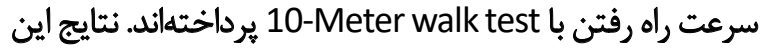

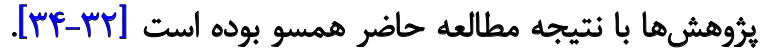

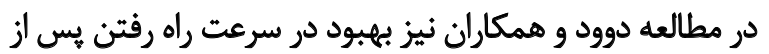

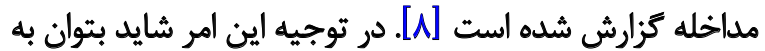

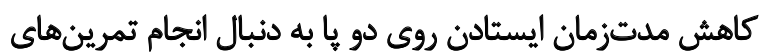

\begin{tabular}{|c|c|c|c|c|}
\hline كودى جههارم & كودى سوم & كودك دوم & كودى اول & اندازه اثر \\
\hline$r / T)$ & $T / N Q$ & $r / T V$ & VIr & Cohen's d \\
\hline V/ar & V/AY & T/q & .194 & Hedges'g \\
\hline
\end{tabular}


كاردرمانى دانشكاه علوم توانبخشى و سلامت اجتماعى انجام شده

مشاركت نويسند انَّان

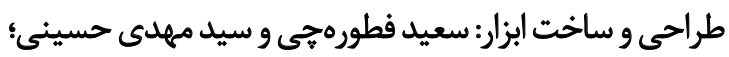

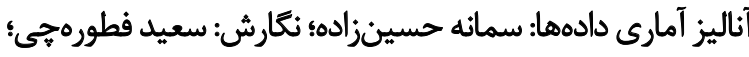
ويراستارى و نظارت: حجتالله حقكو و سيد على حسينى.

$$
\text { تقعارض مثافع }
$$

بنابر اظهار نويسندكان اين مقاله تعارض منافع ندارد.
اشاره كرد. البته ميزان اشتياق و انكيزه اين كودكان در حين

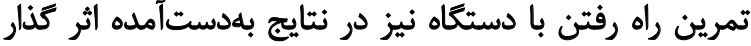

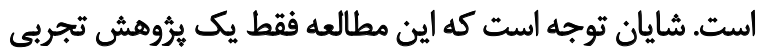

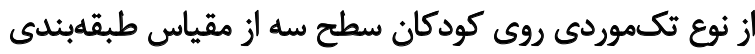

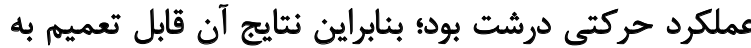

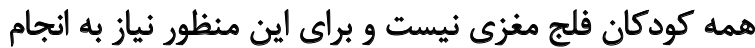

$$
\text { تحقيقات بيشتر است. }
$$

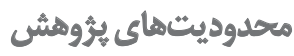

با اينكه سطح عملكرد حركتى درشت كودكان شركت كنينده در

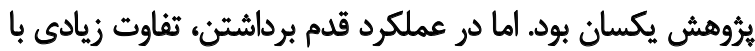

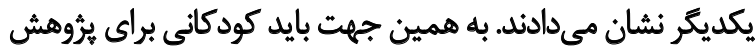

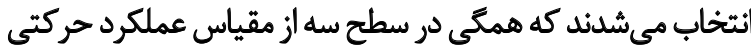

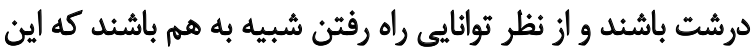

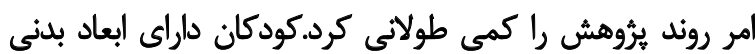

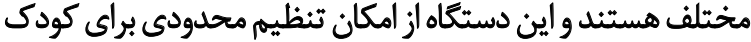

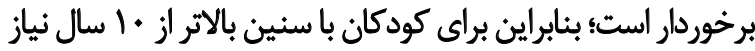

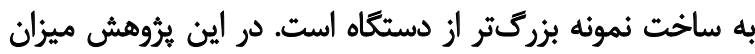

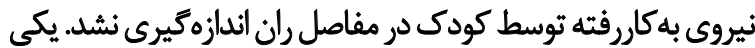

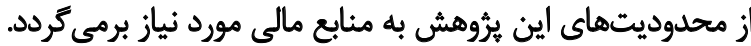

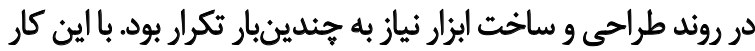

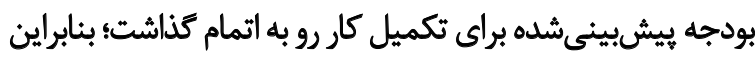

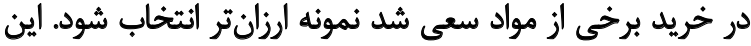

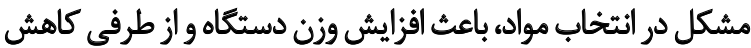

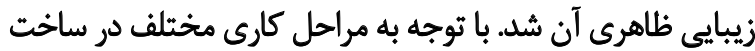

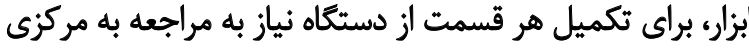

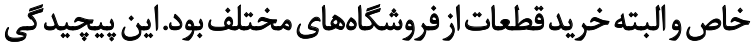

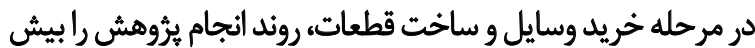

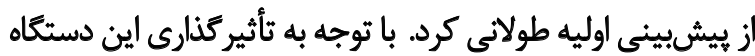

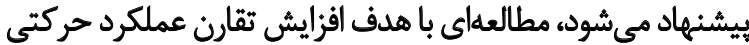

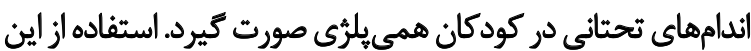

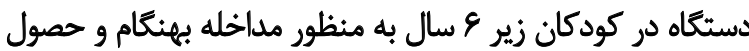
نتايج درمانى بهتر توصيه مي نشودان

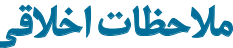

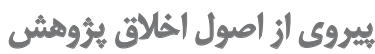

اين يُروهش زير نظر كميته اخلاق در يُؤوهش دانشكاه علوم

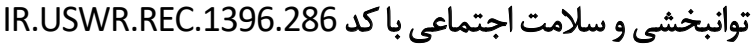

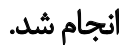

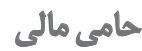

اين يُروهش بخشى از ياياننامه دكتراى نويسنده دوم در گروه 


\section{References}

[1] Stanley FJ, Blair E, Alberman E. Cerebral palsies: Epidemiology and causal pathways. Cambridge: Cambridge University Press; 2000 https://www.google.com/books/edition/Cerebral_Palsies/jEc1q$\mathrm{CNg} 3 \mathrm{IC} ? \mathrm{hl}=\mathrm{en} \& g \mathrm{bpv}=0$

[2] Rosenbaum P, Paneth N, Leviton A, Goldstein M, Bax M, Damiano $\mathrm{D}$, et al. A report: The definition and classification of cerebral palsy April 2006. Developmental medicine and child neurology. Supplement. 2007; 109(suppl 109):8-14. [PMID]

[3] McNevin NH, Coraci L, Schafer J. Gait in adolescent cerebral palsy: the effect of partial unweighting. Archives of Physical Medicine and Rehabilitation. 2000; 81(4):525-8. [DOI:10.1053/mr.2000.4429]

[4] Palisano RJ, Tieman BL, Walter SD, Bartlett DJ, Rosenbaum PL, Russell D, et al. Effect of environmental setting on mobility methods of children with cerebral palsy. Developmental Medicine and Child Neurology. 2003; 45(2):113-20. [DOI:10.1111/j.1469-8749.2003. tb00914.x]

[5] Lancioni GE, Singh NN, O’Reilly MF, Sigafoos J, Didden R, Manfredi F, et al. Fostering locomotor behavior of children with developmental disabilities: An overview of studies using treadmills and walkers with microswitches. Research in Developmental Disabilities. 2009; 30(2):308-22. [DOI:10.1016/j.ridd.2008.05.002] [PMID]

[6] Mcewen IR. Assistive positioning as a control parameter of socialcommunicative interactions between students with profound multiple disabilities and classroom staff. Physical Therapy. 1992; 72: 63447. [DOI:10.1093/pti/72.9.634] [PMID]

[7] Shepherd R. Cerebral palsy. In: Shepherd R, editor. Physiotherapy in paediatrics. Oxford: Butterworth-Heinemann; 1995. https://www. google.com/books/edition/Physiotherapy_in_Paediatrics/WCt2Q gAACAAJ?hl=en\&kptab $=$ overview

[8] Dodd KJ, Foley S. Partial body-weight-supported treadmill training can improve walking in children with cerebral palsy: A clinical controlled trial. Developmental Medicine \& Child Neurology. 2007; 49(2):101-5. [DOI:10.1111/j.1469-8749.2007.00101.x] [PMID]

[9] Scrutton D, Rosenbaum PL. The locomotor devel-opment of children with cerebral palsy. In: Connolly K, Forssberg H, eds.Neurophysiology and Neuropsy-chology of Motor Development. London: Mac Keith Press; 1997. https://www.google.com/books/edition/ Neurophysiology_and_Neuropsychology_of_M/gU6gkdwXR6UC ?hl $=$ en\&gbpv $=0 \& \mathrm{kptab}=$ overview

[10] Rosenbaum PL, Walter SD, Hanna SE, Palisano RJ, Russell DJ, Raina P, et al. Prognosis for gross motor function in cerebral palsy: Creation of motor development curves. JAMA. 2002; 288(11):135763. [DOI:10.1001/jama.288.11.1357] [PMID]

[11] Wood E, Rosenbaum P. The gross motor function classification system for cerebral palsy: A study of reliability and stability over time. Developmental Medicine and Child Neurology. 2000; 42(5):292-6. [DOI:10.1017/S0012162200000529] [PMID]

[12] Liao HF, Jeny SF, Lai JS, Cheng CK, Hu MH. The relation between standing balance and walking function in children with spastic diplegic cerebral palsy. Developmental Medicine \& Child Neurology. 1997; 39(2):106-12. [DOI:10.1111/j.1469-8749.1997.tb07392.x] [PMID]

[13] Andersson C, Mattsson E. Adults with cerebral palsy: A survey describing problems, needs, and resources, with special emphasis on locomotion. Developmental Medicine and Child Neurology. 2001; 43(2):76-82. [DOI:10.1111/j.1469-8749.2001.tb00719.x]

[14] Ostensjø S, Carlberg EB, Vollestad NK. The use and impact of assistive devices and other environmental modifications on everyday activities and care in young children with cerebral palsy. Disability and Rehabilitation. 2005; 27(14):849-61. [DOI:10.1080/09638280400018619] [PMID]

[15] Gorter H, Holty L, Rameckers EE, Elvers HJ, Oostendorp RA. Changes in endurance and walking ability through functional physical training in children with cerebral palsy. Pediatric Physical Therapy. 2009; 21(1):31-7. [DOI:10.1097/PEP.0b013e318196f563] [PMID]

[16] Darrah J, Hickman R, O'donnell M, Vogtle L, Wiart L. AACPDM methodology to develop systematic reviews of treatment interventions (Revision 1.2). Milwaukee, WI, USA: American Academy for Cerebral Palsy and Developmental Medicine; 2008. https://www. aacpdm.org/UserFiles/file/systematic-review-methodology.pdf

[17] Low SA, McCoy SW, Beling J, Adams J. Pediatric physical therapists' use of support walkers for children with disabilities: A nationwide survey. Pediatric Physical Therapy. 2011; 23(4):381-9. [DOI:10.1097/ PEP.0b013e318235257c] [PMID]

[18] Paleg G, Livingstone R. Outcomes of gait trainer use in home and school settings for children with motor impairments: A systematic review. Clinical Rehabilitation. 2015; 29(11):1077-91. [DOI:10.1177/0269215514565947] [PMID]

[19] Nansai S, Elara MR, Iwase M. Dynamic analysis and modeling of Jansen mechanism. Procedia Engineering. 2013; 64:1562-71. [DOI:10.1016/j.proeng.2013.09.238]

[20] Reboussin DM, Morgan TM. Statistical considerations in the use and analysis of single-subject designs. Medicine and Science in Sports and Exercise. 1996; 28(5):639-44. [DOI:10.1249/00005768199605000-00017] [PMID]

[21] Colver A. Study protocol: SPARCLE-a multi-centre European study of the relationship of environment to participation and quality of life in children with cerebral palsy. BMC Public Health. 2006; 6(1):105. [DOI:10.1186/1471-2458-6-105] [PMID] [PMCID]

[22] Alotaibi M, Long T, Kennedy E, Bavishi S. The efficacy of GMFM88 and GMFM- 66 to detect changes in gross motor function in children with cerebral palsy (CP): A literature review. Disability and Rehabilitation. 2014; 36(8):617-27. [DOI:10.3109/09638288.2013.80 5820] [PMID]

[23] Thompson P, Beath T, Bell J, Jacobson G, Phair T, Salbach NM, et al. Test-retest reliability of the 10-metre fast walk test and 6-minute walk test in ambulatory school-aged children with cerebral palsy. Developmental Medicine \& Child Neurology. 2008; 50:370-6. [DOI:10.1111/j.1469-8749.2008.02048.x] [PMID]

[24] Watson MJ. Refining the ten-meter walking test for use with neurologically impaired people. Physiotherapy. 2002; 88(7):386-97. [DOI:10.1016/S0031-9406(05)61264-3]

[25] Begnoche DM, Pitetti KH. Effects of traditional treatment and partial body weight treadmill training on the motor skills of children with spastic cerebral palsy: A pilot study. Pediatric Physical Therapy. 2007; 19(1):11-9. [DOI:10.1097/01.pep.0000250023.06672.b6] [PMID] 
[26] Zhan S, Ottenbacher KJ. Single subject research designs for disability research. Disability and Rehabilitation. 2001; 23(1):1-8. [DOI:10.1080/09638280150211202] [PMID]

[27] Meyer-Heim A, Ammann-Reiffer C, Schmartz A, Schaefer J, Sennhauser FH, Heinen F, et al. Improvement of walking abilities after robotic-assisted locomotion training in children with cerebral palsy. Archives of Disease in Childhood. 2009; 94(8):615-20. [DOI:10.1136/adc.2008.145458] [PMID]

[28] Mattern-Baxter K. Effects of partial body weight supported treadmill training on children with cerebral palsy. Pediatric Physical Therapy. 2009; 21(1):12-22. [DOI:10.1097/ PEP.0b013e318196ef42] [PMID]

[29] Schindl MR, Forstner C, Kern H, Hesse S. Treadmill training with partial body weight support in nonambulatory patients with cerebral palsy. Archives of Physical Medicine and Rehabilitation. 2000; 81(3):301-6. [DOI:10.1016/S0003-9993(00)90075-3] [PMID]

[30] Provost B, Dieruf K, Burtner PA, Phillips JP, BernitskyBeddingfield A, Sullivan KJ, et al. Endurance and gait in children with cerebral palsy after intensive body weight supported treadmill training. Pediatric Physical Therapy. 2007; 19(1):2-10. [DOI:10.1097/01.pep.0000249418.25913.a3] [PMID]

[31] Eisenberg S, Zuk L, Carmeli E, Katz-Leurer M. Contribution of stepping while standing to function and secondary conditions among children with cerebral palsy. Pediatric Physical Therapy. 2009; 21(1):79-85. [DOI:10.1097/PEP.0b013e31818f57f2] [PMID]

[32] Banz R, Bolliger M, Colombo G, Dietz V, Lünenburger L. Computerized visual feedback: an adjunct to robotic-assisted gait training. Physical Therapy. 2008; 88(10):1135-45. [DOI:10.2522/ ptj.20070203] [PMID]

[33] Cho C, Hwang W, Hwang S, Chung Y. Treadmill training with virtual reality improves gait, balance, and muscle strength in children with cerebral palsy. The Tohoku Journal of Experimental Medicine. 2016; 238(3):213-8. [DOI:10.1620/tjem.238.213] [PMID]

[34] Swe NN, Sendhilnnathan S, van Den Berg M, Barr C. Over ground walking and body weight supported walking improve mobility equally in cerebral palsy: A randomised controlled trial. Clinical Rehabilitation. 2015; 29(11):1108-16. [DOI:10.1177/0269215514566249] [PMID]

[35] Cherng RJ, Liu CF, Lau TW, Hong RB. Effect of treadmill training with body weight support on gait and gross motor function in children with spastic cerebral palsy. American Journal of Physical Medicine \& Rehabilitation. 2007; 86(7):548-55. [DOI:10.1097/ PHM.0b013e31806dc302] [PMID]

[36] Wang X, Wang Y. Gait analysis of children with spastic hemiplegic cerebral palsy. Neural Regeneration Research. 2012; 7(20):1578-84. [DOI:10.3969/j.issn.1673-5374.2012.20.008] [PMID]

[37] Willoughby KL, Dodd KJ, Shields N, Foley S. Efficacy of partial body weight-supported treadmill training compared with overground walking practice for children with cerebral palsy: A randomized controlled trial. Archives of Physical Medicine and Rehabilitation. 2010; 91(3):333-9. [DOI:10.1016/j.apmr.2009.10.029] [PMID]
[38] Fatorehchy S, Hosseini SA, Rassafiani M. The effect of aquatic therapy at different levels of water depth on functional balance and walking capacity in children with cerebral. International Journal of Life Science and Pharma Research. 2019; 9(1):L52-L57. [DOI:10.22376/ijpbs/lpr.2019.9.1.L52-57]

[39] Fatorehchy S, Hosseini SA, Haghgoo HA, Hosseinzadeh S. The effect of gait enhancer mechanism on functional balance and endurance of walking in children with cerebral palsy. Medical Science. 2019; 23(99):724-31. https://www.semanticscholar. org/paper/The-effect-of-gait-enhancer-mechanism-on-functional-Fatorehchy-Hosseini/0700fe27f2052e361ae5436c82a856 5e5b0c4a03 
This Page Intentionally Left Blank 